\title{
Phase Equilibria in the System Niobium Pentoxide-- Boric Acid
}

\author{
Ernest M. Levin
}

(October 11, 1965)

\begin{abstract}
The phase-equilibrium diagram for the system $\mathrm{Nb}_{2} \mathrm{O}_{5}-\mathrm{B}_{2} \mathrm{O}_{3}$ was determined experimentally using the quenching technique and examining the samples by optical microscopy and x-ray powder diffractometry. The system contains one binary compound of approximate composition " $3 \mathrm{Nb}_{2} \mathrm{O}_{5}$. $\mathrm{B}_{2} \mathrm{O}_{3}$," which melts incongruently at about $1150{ }^{\circ} \mathrm{C}$ to $\mathrm{H}-\mathrm{Nb}_{2} \mathrm{O}_{5}$ and $\mathrm{B}_{2} \mathrm{O}_{3}$-rich liquid. A large region of liquid immiscibility extends, at $1352{ }^{\circ} \mathrm{C}$, from 10 mole percent $\mathrm{Nb}_{2} \mathrm{O}_{5}$ to 65.7 mole percent $\mathrm{Nb}_{2} \mathrm{O}_{5}$. The theoretically calculated extent of immiscibility is in reasonable agreement with the experimentally determined value.
\end{abstract}

Key Words: Boric oxide, immiscibility, niobium pentoxide, oxides, phase equilibria.

\section{Introduction}

Consistent with the objective expressed in the Introduction to the preceding paper on the system $\mathrm{Nb}_{2} \mathrm{O}_{5}-$ $\mathrm{GeO}_{2}[1],{ }^{1}$ the systematic study of immiscibility was extended to include the phase relations in the system $\mathrm{Nb}_{2} \mathrm{O}_{5}-\mathrm{B}_{2} \mathrm{O}_{3}$. The $\mathrm{Nb}^{5+}$ cation has a high charge and moderate ionic radius, $0.69 \AA$; and, consequently, a high ionic field strength. Notwithstanding the high field strength, the $\mathrm{Nb}_{2} \mathrm{O}_{5}-\mathrm{GeO}_{2}$ system does not show liquid immiscibility. However, the $\mathrm{Nb}_{2} \mathrm{O}_{5}-\mathrm{SiO}_{2}$ system was found to contain a large region of immiscibility [2]. Based on the observation that no case has been reported in which liquid immiscibility exists for the silicate system but not for the corresponding borate system, immiscibility in the $\mathrm{Nb}_{2} \mathrm{O}_{5}-\mathrm{B}_{2} \mathrm{O}_{3}$ system was to be expected. Furthermore, the system offered a possible opportunity to apply and to extend the principles of immiscibility developed mainly from systems of borates with the oxides of divalent cations.

\section{Sample Preparation and Test Methods}

Starting materials for the preparation of mixtures consisted of high-purity niobium pentoxide and reagent grade boric acid powder. The $\mathrm{Nb}_{2} \mathrm{O}_{5}$ contained the following impurities when examined by the general qualitative spectrochemical method: $\mathrm{Si}-$ less than 0.1 percent; $\mathrm{Fe}, \mathrm{Sn}, \mathrm{Ti}-0.001$ to 0.01 percent; $\mathrm{Ca}, \mathrm{Mg}-$ 0.0001 to 0.001 percent; $\mathrm{Cu}-$ ? The boric acid was of especially high purity, containing only faint traces $(0.0001$ to $0.001 \%)$ of $\mathrm{Mg}$ and $\mathrm{Si}$.

Three-gram batches, on an ignited basis, of the starting materials were formulated by mixing or grinding, pressing, and heating for $4 \mathrm{hr}$, at three successively higher temperatures: 700,800 , and about $900{ }^{\circ} \mathrm{C}$. Phase equilibrium relations were studied by the wellestablished quenching technique. Samples were heated in sealed $\mathrm{Pt}$ tubes for periods of 1 to $336 \mathrm{hr}$.

' Figures in brackets indicate the literature references at the end of this paper.
Temperatures were measured with a calibrated Pt90Pt:10 Rh thermocouple. Quenched samples were examined with the binocular and polarizing microscopes and by x-ray powder diffractometry (Ni-filtered $\mathrm{CuK}_{\alpha}$ radiation) using a high-angle Geiger-counter diffractometer. The technique and details of sample preparation, apparatus, and method have been described in previous publications [3] and were the same as for the study of the $\mathrm{Nb}_{2} \mathrm{O}_{5}-\mathrm{GeO}_{2}$ system [1]. The overall maximum uncertainty of the liquidus temperatures reported are estimated to be within $\pm 10{ }^{\circ} \mathrm{C}$.

It should be noted that the polarizing microscope was of limited value because clear homogeneous glass was not observed, even for the compositions richest in $\mathrm{B}_{2} \mathrm{O}_{3}$. Quenched liquid devitrified, to lesser or greater extent, and the grains appeared brownish, finely mottled, and very weakly birefringent, when viewed under the microscope.

\section{Chemical Analysis}

Formulated compositions were analyzed by the Analytical Chemistry Division. For most of the compositions only the $\mathrm{Nb}_{2} \mathrm{O}_{5}$ content was determined, by titration of an unbuffed solution at $p \mathrm{H} 4.5$ with standard EDTA solution. Percent $\mathrm{B}_{2} \mathrm{O}_{3}$ was estimated by difference from 100 percent. Analyses of three compositions (formulated at 25,50, and 66.7 mole percent $\mathrm{Nb}_{2} \mathrm{O}_{5}$ ) as well as analysis of the mechanically separated heavy and light devitrified liquids in the immiscibility region were made for both $\mathrm{Nb}_{2} \mathrm{O}_{5}$ and $\mathrm{B}_{2} \mathrm{O}_{3}$. $\mathrm{B}_{2} \mathrm{O}_{3}$ was determined by titration of a neutral solution $(p \mathrm{H} 7.00)$ with standard $\mathrm{NaOH}$ solution, after the additions of manitol. $\mathrm{Nb}_{2} \mathrm{O}_{5}$ was determined from the weight of the ignited residue. Analyzed values were used in constructing the phase diagram. Those compositions for which one of the components was determined by difference have an estimated uncertainty of about 2 mole percent. Those for which both components were analyzed have an estimated accuracy of better than 1 mole percent. 


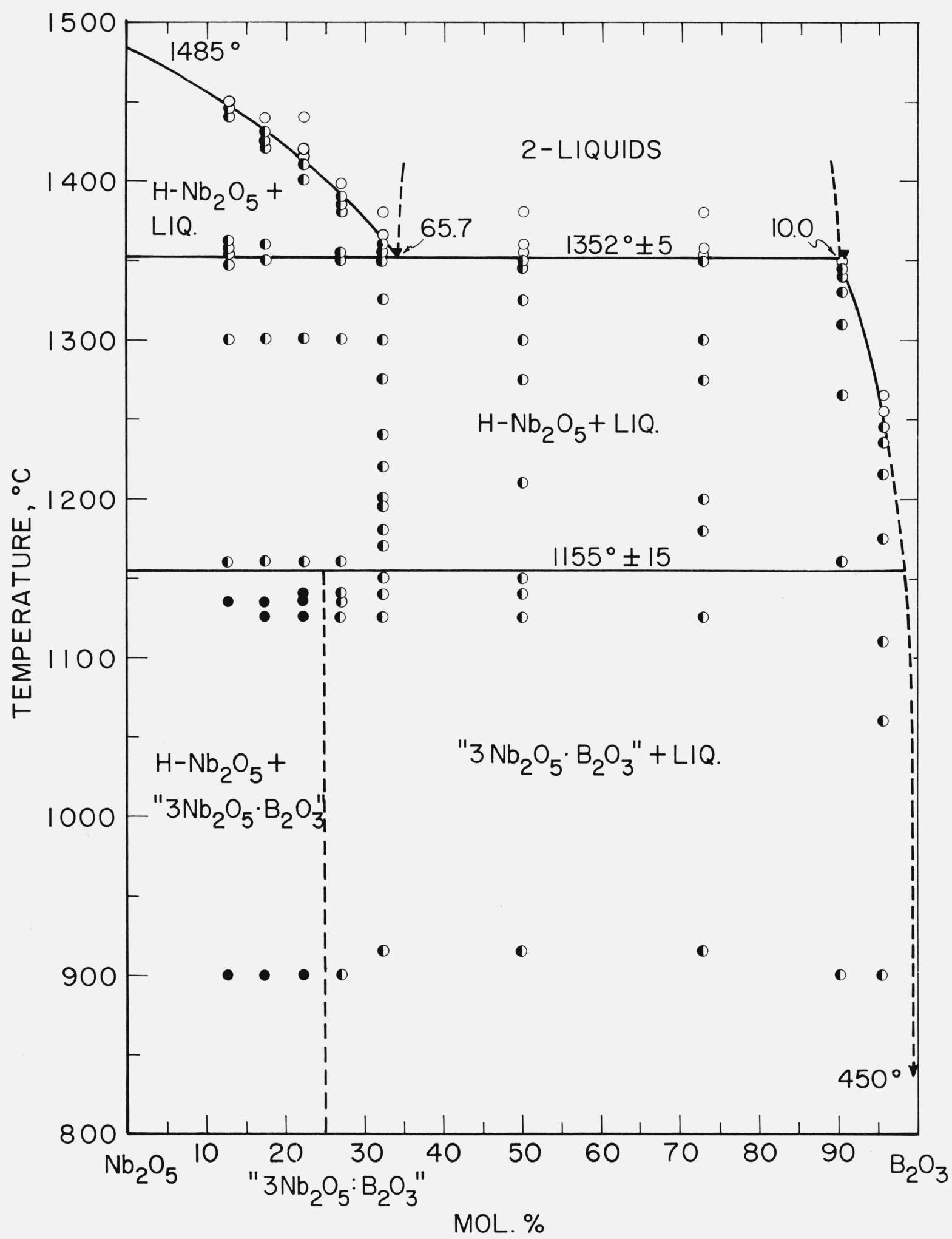

Figure 1. Phase diagram for the system $\mathrm{Nb}_{2} \mathrm{O}_{5}-\mathrm{B}_{2} \mathrm{O}_{3}$.

- No melting; $\bigcirc$-partial melting; $\bigcirc$-complete melting;

chemically analyzed value of mechanically separated phases in 


\section{Results and Discussion}

\section{1. $\mathrm{Nb}_{2} \mathrm{O}_{5}$ and $\mathrm{B}_{2} \mathrm{O}_{3}$ Components}

No quenching experiments were made with the components, as they were the same materials that had been used previously in phase equilibrium studies originating in this laboratory $[3,4]$. As regards the $\mathrm{Nb}_{2} \mathrm{O}_{5}$ component, the same remarks pertain as for the $\mathrm{Nb}_{2} \mathrm{O}_{5}-\mathrm{GeO}_{2}$ system [1]. For purposes of consistency with previous publications the indicated melting temperature of $\mathrm{B}_{2} \mathrm{O}_{3}$ is given as $450{ }^{\circ} \mathrm{C}$, although this value may be $5^{\circ}$ to $10^{\circ}$ low [5].

\subsection{Phase Diagram}

The phase diagram for the system is shown in figure 1 , and table 1 lists the compositions studied, heat treatments, and phases identified. The system is characterised by a large region of liquid immiscibility, extending from about 10 mole percent to 65.7 mole percent $\mathrm{Nb}_{2} \mathrm{O}_{5}$, and by a binary compound, " $3 \mathrm{Nb}_{2} \mathrm{O}_{5}$. $\mathrm{B}_{2} \mathrm{O}_{3}$ " melting incongruently at $1150{ }^{\circ} \mathrm{C}$, some $200^{\circ}$ below the monotectic.

The two triangles in figure 1 designate the chemically analyzed values for the $\mathrm{B}_{2} \mathrm{O}_{3}$-rich and the $\mathrm{Nb}_{2} \mathrm{O}_{5}$ -

TABLE 1. Experimental data for compositions in the binary system $\mathrm{Nb}_{2} \mathrm{O}_{5}-\mathrm{B}_{2} \mathrm{O}_{3}$

\begin{tabular}{|c|c|c|c|c|c|c|}
\hline \multicolumn{2}{|c|}{ Composition $^{1}$} & \multicolumn{2}{|c|}{ Heat treatment ${ }^{2}$} & \multicolumn{2}{|r|}{ Results } & \multirow{2}{*}{ Notes } \\
\hline $\mathrm{Nb}_{2} \mathrm{O}_{5}$ & $\mathrm{~B}_{2} \mathrm{O}_{3}$ & Temp. & Time & Physical observation & X-ray diffraction analyses ${ }^{3}$ & \\
\hline $\begin{array}{r}\text { Mole \% } \\
4.4\end{array}$ & $\begin{array}{c}\text { Mole \% } \\
95.6\end{array}$ & $\begin{array}{c}{ }^{\circ} \mathrm{C} \\
900 \\
1061 \\
1109 \\
1175 \\
1216 \\
1235 \\
1246 \\
1256 \\
1265\end{array}$ & $\begin{array}{l}\text { Hour } \\
\quad 3 \\
1 \\
1.5 \\
1 \\
1 \\
2 \\
2 \\
2 \\
2\end{array}$ & $\begin{array}{l}\text { Considerable melting. } \\
\text { Considerable melting. } \\
\text { Considerable melting. } \\
\text { Considerable melting. } \\
\text { Considerable melting. } \\
\text { Considerable melting. } \\
\text { Near complete melting. } \\
\text { Complete melting. } \\
\text { Complete melting. }\end{array}$ & $\begin{array}{l}3: 1+\text { glass hump }+\left[\mathrm{H}_{3} \mathrm{BO}_{3}\right] \\
3: 1+\text { glass hump }+\left[\mathrm{L}-\mathrm{Nb}_{2} \mathrm{O}_{5}\right] \\
3: 1+\text { glass hump }+\left[\mathrm{L}-\mathrm{Nb}_{2} \mathrm{O}_{5}\right] \\
{\left[\mathrm{H}_{3} \mathrm{BO}_{3}\right]+3: 1+\left[\mathrm{L}-\mathrm{Nb}_{2} \mathrm{O}_{5}\right]+\mathrm{H}-\mathrm{Nb}_{2} \mathrm{O}_{5}} \\
{\left[\mathrm{~L}-\mathrm{Nb}_{2} \mathrm{O}_{5}\right]+3: 1+\left[\mathrm{H}_{3} \mathrm{BO}_{3}\right]+\mathrm{H}-\mathrm{Nb}_{2} \mathrm{O}_{5}} \\
3: 1+\left[\mathrm{H}_{3} \mathrm{BO}_{3}\right]+\left[\mathrm{L}-\mathrm{Nb}_{2} \mathrm{O}_{5}\right]+\mathrm{H}-\mathrm{Nb}_{2} \mathrm{O}_{5} \\
{\left[\mathrm{~L}-\mathrm{Nb}_{2} \mathrm{O}_{5}\right]+[3: 1]} \\
{\left[\mathrm{L}-\mathrm{Nb}_{2} \mathrm{O}_{5}\right]+[3: 1]}\end{array}$ & $\begin{array}{l}\mathrm{No} \mathrm{H}-\mathrm{Nb}_{2} \mathrm{O}_{5} \text { detected. } \\
\text { Tube leaked. }\end{array}$ \\
\hline 9.7 & 90.3 & $\begin{array}{r}900 \\
1161 \\
1265 \\
1310 \\
1331 \\
1339 \\
1345 \\
1350\end{array}$ & $\begin{array}{c}3 \\
68 \\
2 \\
1.5 \\
1.5 \\
1.5 \\
1.5 \\
1.5\end{array}$ & $\begin{array}{l}\text { Some melting. } \\
\text { Considerable melting. } \\
\text { Considerable melting. } \\
\text { Considerable melting. } \\
\text { Considerable melting. } \\
\text { Considerable melting. } \\
\text { Near complete melting. } \\
\text { Complete melting. }\end{array}$ & $\begin{array}{l}3: 1+\text { glass hump } \\
3: 1+\mathrm{H}-\mathrm{Nb}_{2} \mathrm{O}_{5} \\
{\left[\mathrm{~L}-\mathrm{Nb}_{2} \mathrm{O}_{5}\right]+3: 1} \\
3: 1+\left[\mathrm{L}-\mathrm{Nb}_{2} \mathrm{O}_{5}\right]+\mathrm{H}-\mathrm{Nb}_{2} \mathrm{O}_{5} \\
3: 1+\left[\mathrm{L}-\mathrm{Nb}_{2} \mathrm{O}_{5}\right]+\mathrm{H}-\mathrm{Nb}_{2} \mathrm{O}_{5} \\
3: 1+\left[\mathrm{L}-\mathrm{Nb}_{2} \mathrm{O}_{5}\right]+\left[\mathrm{H}_{3} \mathrm{BO}_{3}\right] \\
{\left[\mathrm{L}-\mathrm{Nb}_{2} \mathrm{O}_{5}\right]+3: 1+\mathrm{H}-\mathrm{Nb}_{2} \mathrm{O}_{5}} \\
{\left[\mathrm{~L}-\mathrm{Nb}_{2} \mathrm{O}_{5}\right]}\end{array}$ & $\begin{array}{l}\text { Above decomp. } 3: 1 . \\
\text { Very weak x-ray pattern. } \\
\text { Tube leaked. } \\
\text { One liquid. }\end{array}$ \\
\hline 27.2 & 72.8 & $\begin{array}{r}915 \\
1180 \\
1200 \\
1275 \\
1300 \\
1300 \\
1125 \\
1349 \\
1354 \\
1357 \\
1380\end{array}$ & $\begin{array}{c}4 \\
53.5 \\
66.5 \\
15 \\
18 \\
18 \\
117\} \\
2 \\
1.5 \\
1 \\
2\end{array}$ & $\begin{array}{l}\text { Slight melting. } \\
\text { Some melting. } \\
\text { Some melting. } \\
\text { Some melting. } \\
\text { Some melting. } \\
\\
\text { Some melting. } \\
\text { Complete melting. } \\
\text { Complete melting. } \\
\text { Complete melting. }\end{array}$ & $\begin{array}{l}3: 1 \\
3: 1+\mathrm{H}-\mathrm{Nb}_{2} \mathrm{O}_{5} \\
3: 1+\mathrm{H}-\mathrm{Nb}_{2} \mathrm{O}_{5} \\
3: 1+\mathrm{H}-\mathrm{Nb}_{2} \mathrm{O}_{5} \\
\mathrm{H}-\mathrm{Nb}_{2} \mathrm{O}_{5}+3: 1+\left[\mathrm{L}-\mathrm{Nb}_{2} \mathrm{O}_{5}\right] \\
3: 1+\mathrm{H}-\mathrm{Nb}_{2} \mathrm{O}_{5} \\
\mathrm{H}-\mathrm{Nb}_{2} \mathrm{O}_{5}+\left[\mathrm{L}-\mathrm{Nb}_{2} \mathrm{O}_{5}\right]+3: 1 \\
{\left[\mathrm{~L}-\mathrm{Nb}_{2} \mathrm{O}_{5}\right]+\left[\mathrm{H}-\mathrm{Nb}_{2} \mathrm{O}_{5}\right]}\end{array}$ & $\begin{array}{l}\left\{\begin{array}{l}\text { Shows reversibility: } \\
3: 1 \leftrightarrow \mathrm{H}-\mathrm{Nb}_{2} \mathrm{O}_{5}+\text { liquid. }\end{array}\right. \\
\text { Above monotectic. } \\
\text { Chem. Analysis of each phase. }\end{array}$ \\
\hline 50.2 & 49.8 & $\begin{array}{r}915 \\
1140 \\
1150 \\
1210 \\
1275 \\
1300 \\
1300 \\
1125 \\
1325 \\
1345 \\
1350 \\
1356 \\
1360 \\
1380\end{array}$ & $\begin{array}{r}4 \\
69 \\
45 \\
40 \\
15 \\
18 \\
18 \\
117\} \\
1 \\
1.25 \\
1 \\
1.5 \\
1.25 \\
2\end{array}$ & $\begin{array}{l}\text { Slight melting. } \\
\text { Slight melting. } \\
\text { Some melting. } \\
\text { Some melting. } \\
\text { Some melting. } \\
\text { Considerable melting. } \\
\\
\text { Considerable melting. } \\
\text { Considerable melting. } \\
\text { Considerable melting. } \\
\text { Complete melting. } \\
\text { Complete melting. } \\
\text { Complete melting. }\end{array}$ & $\begin{array}{l}3: 1 \\
3: 1+\left[\mathrm{H}_{3} \mathrm{BO}_{3}\right] \\
3: 1 \\
3: 1+\mathrm{H}-\mathrm{Nb}_{2} \mathrm{O}_{5} \\
\mathrm{H}-\mathrm{Nb}_{2} \mathrm{O}_{5}+3: 1 \\
\mathrm{H}-\mathrm{Nb}_{2} \mathrm{O}_{5}+3: 1 \\
3: 1+\mathrm{H}-\mathrm{Nb}_{2} \mathrm{O}_{5} \\
3: 1+\mathrm{H}-\mathrm{Nb}_{2} \mathrm{O}_{5} \\
3: 1+\mathrm{H}-\mathrm{Nb}_{2} \mathrm{O}_{5} \\
\left.\mathrm{H}-\mathrm{Nb}_{2} \mathrm{O}_{5}+\left[\mathrm{L}-\mathrm{Nb}_{2} \mathrm{O}_{5}\right]+3:\right] \\
{\left[\mathrm{H}-\mathrm{Nb}_{2} \mathrm{O}_{5}\right]+\left[\mathrm{L}-\mathrm{Nb}_{2} \mathrm{O}_{5}\right]}\end{array}$ & $\begin{array}{l}\text { Above decomp. } 3: 1 . \\
\left\{\begin{array}{l}\text { Shows reversibility: } \\
3: 1 \leftrightarrow \mathrm{H}-\mathrm{Nb}_{2} \mathrm{O}_{5}+\text { liquid. }\end{array}\right. \\
\text { Decomp. } 3: 1 \text { sluggish. } \\
\text { Above monotectic. } \\
\text { For chem. analysis. } \\
\text { Above monotectic. }\end{array}$ \\
\hline 67.8 & 32.2 & $\begin{array}{r}915 \\
1140 \\
1150 \\
1170 \\
1180 \\
1194 \\
1200 \\
1220 \\
1240 \\
1275 \\
1300 \\
1300 \\
1125 \\
1325 \\
1350 \\
1356 \\
1361 \\
1367 \\
1380\end{array}$ & $\begin{array}{c}4 \\
69 \\
45 \\
20 \\
53.5 \\
1.75 \\
66.5 \\
8.5 \\
1.5 \\
1.5 \\
18 \\
18 \\
117 \\
1 \\
1 \\
1 \\
1.5 \\
1 \\
2\end{array}$ & $\begin{array}{l}\text { Slight melting. } \\
\text { Slight melting. } \\
\text { Slight melting. } \\
\text { Melting. } \\
\text { Melting. } \\
\text { Melting. } \\
\text { Melting. } \\
\text { Melting. } \\
\text { Melting. } \\
\text { Melting. } \\
\text { Melting. }\end{array}$ & $\begin{array}{l}3: 1 \\
3: 1 \\
3: 1 \\
3: 1+\mathrm{H}-\mathrm{Nb}_{2} \mathrm{O}_{5}(?) \\
3: 1+\mathrm{H}-\mathrm{Nb}_{2} \mathrm{O}_{5} \\
3: 1 \\
3: 1+\mathrm{H}-\mathrm{Nb}_{2} \mathrm{O}_{5} \\
3: 1+\mathrm{H}-\mathrm{Nb}_{2} \mathrm{O}_{5} \\
3: 1+\mathrm{H}-\mathrm{Nb}_{2} \mathrm{O}_{5} \\
\mathrm{H}-\mathrm{Nb}_{2} \mathrm{O}_{5}+3: 1 \\
\mathrm{H}-\mathrm{Nb}_{2} \mathrm{O}_{5}+3: 1(?) \\
3: 1+\mathrm{H}-\mathrm{Nb}_{2} \mathrm{O}_{5} \\
3: 1+\mathrm{H}-\mathrm{Nb}_{2} \mathrm{O}_{5} \\
\mathrm{H}-\mathrm{Nb}_{2} \mathrm{O}_{5}+\left[\mathrm{L}-\mathrm{Nb}_{2} \mathrm{O}_{5}\right] \\
\mathrm{H}-\mathrm{Nb}_{2} \mathrm{O}_{5} \\
\mathrm{H}-\mathrm{Nb}_{2} \mathrm{O}_{5}+[3: 1] \\
{\left[\mathrm{L}-\mathrm{Nb}_{2} \mathrm{O}_{5}\right]+[3: 1]} \\
{\left[\mathrm{L}-\mathrm{Nb}_{2} \mathrm{O}_{5}\right]+[3: 1]}\end{array}$ & $\begin{array}{l}\left\{\begin{array}{l}\text { Shows reversibility: } \\
3: 1 \leftrightarrow \mathrm{H}-\mathrm{Nb}_{2} \mathrm{O}_{5}+\text { liquid. }\end{array}\right. \\
\text { Above monotectic. } \\
\text { One liquid. }\end{array}$ \\
\hline
\end{tabular}


TABLE 1. Experimental data for compositions in the binary system $\mathrm{Nb}_{2} \mathrm{O}_{5}-\mathrm{B}_{2} \mathrm{O}_{3}-$ Continued

\begin{tabular}{|c|c|c|c|c|c|c|}
\hline \multicolumn{2}{|c|}{ Composition $^{1}$} & \multicolumn{2}{|c|}{ Heat treatment ${ }^{2}$} & \multirow[b]{2}{*}{ Physical observation } & \multirow{2}{*}{$\begin{array}{l}\text { Results } \\
\qquad \text { X-ray diffraction analyses }{ }^{3}\end{array}$} & \multirow[b]{2}{*}{ Notes } \\
\hline $\mathrm{Nb}_{2} \mathrm{O}_{5}$ & $\mathrm{~B}_{2} \mathrm{O}_{5}$ & Temp. & Time & & & \\
\hline Mole \% & Mole \% & ${ }^{\circ} \mathrm{C}$ & Hour & & & \\
\hline 73.0 & 27.0 & $\begin{array}{r}900 \\
1135 \\
1140 \\
1161 \\
1300 \\
1300 \\
1125 \\
1351 \\
1355 \\
1380 \\
1385 \\
1390 \\
1398\end{array}$ & $\begin{array}{r}3 \\
336 \\
69 \\
68 \\
18 \\
18 \\
117\} \\
1.5 \\
1.5 \\
1.5 \\
1.5 \\
1.5 \\
1.5\end{array}$ & $\begin{array}{l}\text { Opalescent. } \\
\text { Opalescent. } \\
\text { Opalescent. } \\
\text { Opalescent. } \\
\text { Opalescent. } \\
\\
\text { Slight melting. } \\
\text { Melting. } \\
\text { Considerable melting. } \\
\text { Near complete melting. } \\
\text { Near complete melting. } \\
\text { Complete melting. }\end{array}$ & $\begin{array}{l}3: 1 \\
3: 1 \\
3: 1 \\
3: 1+\mathrm{H}-\mathrm{Nb}_{2} \mathrm{O}_{5} \\
\mathrm{H}-\mathrm{Nb}_{2} \mathrm{O}_{5}+3: 1 \text { (?) } \\
\mathrm{H}-\mathrm{Nb}_{2} \mathrm{O}_{5}+3: 1 \\
\mathrm{H}-\mathrm{Nb}_{2} \mathrm{O}_{5} \\
\mathrm{H}-\mathrm{Nb}_{2} \mathrm{O}_{5}+3: 1 \\
\mathrm{H}-\mathrm{Nb}_{2} \mathrm{O}_{5}+3: 1 \\
\mathrm{H}-\mathrm{Nb}_{2} \mathrm{O}_{5}+\left[\mathrm{L}-\mathrm{Nb}_{2} \mathrm{O}_{5}\right]+3: 1 \\
{\left[\mathrm{~L}-\mathrm{Nb}_{2} \mathrm{O}_{5}\right]+\mathrm{H}-\mathrm{Nb}_{2} \mathrm{O}_{5}+3: 1} \\
{\left[\mathrm{H}-\mathrm{Nb}_{2} \mathrm{O}_{5}\right]+\left[\mathrm{L}-\mathrm{Nb}_{2} \mathrm{O}_{5}\right]+[3: 1]}\end{array}$ & $\begin{array}{l}\text { Above decomp. } 3: 1 . \\
\left\{\begin{array}{l}\text { Shows reversibility: } \\
3: 1 \leftrightarrow \mathrm{H}-\mathrm{Nb}_{2} \mathrm{O}_{5}+\text { liquid. }\end{array}\right. \\
\text { Above monotectic. } \\
\text { All devitrify from liquid. }\end{array}$ \\
\hline 77.8 & 22.2 & $\begin{array}{r}900 \\
1135 \\
1140 \\
1161 \\
1300 \\
1300 \\
1125 \\
1401 \\
1409 \\
1415 \\
1420 \\
1440\end{array}$ & $\begin{array}{r}3 \\
336 \\
69 \\
68 \\
18 \\
18 \\
117 \\
1 \\
2.5 \\
1.5 \\
1 \\
1\end{array}$ & $\begin{array}{l}\text { No melting. } \\
\text { No melting. } \\
\text { No melting. } \\
\text { Slight melting. } \\
\text { Opalescent. } \\
\\
\text { Considerable melting. } \\
\text { Near complete melting. } \\
\text { Complete melting. } \\
\text { Complete melting. } \\
\text { Complete melting. }\end{array}$ & $\begin{array}{l}3: 1 \\
3: 1 \\
3: 1 \\
3: 1+\mathrm{H}-\mathrm{Nb}_{2} \mathrm{O}_{5} \\
\mathrm{H}-\mathrm{Nb}_{2} \mathrm{O}_{5}+3: 1(?) \\
\mathrm{H}-\mathrm{Nb}_{2} \mathrm{O}_{5}+3: 1 \\
\mathrm{H}-\mathrm{Nb}_{2} \mathrm{O}_{5}+\left[\mathrm{L}-\mathrm{Nb}_{2} \mathrm{O}_{5}\right]+3: 1 \\
\mathrm{H}-\mathrm{Nb}_{2} \mathrm{O}_{5}+\left[\mathrm{L}-\mathrm{Nb}_{2} \mathrm{O}_{5}\right]+3: 1 \\
{\left[\mathrm{H}-\mathrm{Nb}_{2} \mathrm{O}_{5}\right]+\left[\mathrm{L}-\mathrm{Nb}_{2} \mathrm{O}_{5}\right]+[3: 1]} \\
{\left[\mathrm{H}-\mathrm{Nb}_{2} \mathrm{O}_{5}\right]+\left[\mathrm{L}-\mathrm{Nb}_{2} \mathrm{O}_{5}\right]} \\
{\left[\mathrm{L}-\mathrm{Nb}_{2} \mathrm{O}_{5}\right]+\left[\mathrm{H}-\mathrm{Nb}_{2} \mathrm{O}_{5}\right]}\end{array}$ & $\begin{array}{l}\text { Above decomp. } 3: 1 . \\
\left\{\begin{array}{l}\text { Shows reversibility: } \\
3: 1 \leftrightarrow \mathrm{H}-\mathrm{Nb}_{2} \mathrm{O}_{5}+\text { liquid. }\end{array}\right.\end{array}$ \\
\hline 82.9 & 17.1 & $\begin{array}{r}900 \\
1135 \\
1161 \\
1300 \\
1300 \\
1125 \\
1349 \\
1359 \\
1420 \\
1425 \\
1430 \\
1440\end{array}$ & $\begin{array}{r}3 \\
336 \\
68 \\
18 \\
18 \\
117 \\
3 \\
3 \\
1 \\
1.5 \\
1 \\
1\end{array}$ & $\begin{array}{l}\text { No melting. } \\
\text { No melting. } \\
\text { Slight melting. } \\
\text { Melting. } \\
\text { Melting. } \\
\text { Considerable melting. } \\
\text { Considerable melting. } \\
\text { Near complete melting. } \\
\text { At liquidus. } \\
\text { Complete melting. }\end{array}$ & $\begin{array}{l}3: 1 \\
3: 1+\mathrm{H}-\mathrm{Nb}_{2} \mathrm{O}_{5} \\
3: 1+\mathrm{H}-\mathrm{Nb}_{2} \mathrm{O}_{5} \\
\mathrm{H}-\mathrm{Nb}_{2} \mathrm{O}_{5}+3: 1(?) \\
\mathrm{H}-\mathrm{Nb}_{2} \mathrm{O}_{5}+3: 1 \\
\mathrm{H}-\mathrm{Nb}_{2} \mathrm{O}_{5}+\left[\mathrm{L}-\mathrm{Nb}_{2} \mathrm{O}_{5}\right] \\
\mathrm{H}-\mathrm{Nb}_{2} \mathrm{O}_{5}+\left[\mathrm{L}-\mathrm{Nb}_{2} \mathrm{O}_{5}\right] \\
\mathrm{H}-\mathrm{Nb}_{2} \mathrm{O}_{5}+\left[\mathrm{L}-\mathrm{Nb}_{2} \mathrm{O}_{5}\right]+3: 1 \\
\mathrm{H}-\mathrm{Nb}_{2} \mathrm{O}_{5}+3: 1(?) \\
{\left[\mathrm{H}-\mathrm{Nb}_{2} \mathrm{O}_{5}\right]+\left[\mathrm{L}-\mathrm{Nb}_{2} \mathrm{O}_{5}\right]+[3: 1(?)]} \\
{\left[\mathrm{H}-\mathrm{Nb}_{2} \mathrm{O}_{5}\right]+\left[\mathrm{L}-\mathrm{Nb}_{2} \mathrm{O}_{5}\right]}\end{array}$ & $\begin{array}{l}\text { Above decomp. } 3: 1 . \\
\text { Reversibility sluggish. } \\
\text { Below monotectic. } \\
\text { Above monotectic. }\end{array}$ \\
\hline 87.2 & 12.8 & $\begin{array}{r}900 \\
1135 \\
1161 \\
1300 \\
1348 \\
1356 \\
1358 \\
1362 \\
1440 \\
1445 \\
1450\end{array}$ & $\begin{array}{c}3 \\
336 \\
68 \\
18 \\
2 \\
2 \\
1.5 \\
1.5 \\
1 \\
1 \\
1\end{array}$ & $\begin{array}{l}\text { No melting. } \\
\text { No melting. } \\
\text { Melting. } \\
\text { Melting. } \\
\text { Melting. } \\
\text { Moderate melting. } \\
\text { Moderate melting. } \\
\text { Moderate melting. } \\
\text { Considerable melting. } \\
\text { Near complete melting. } \\
\text { Complete melting. }\end{array}$ & $\begin{array}{l}3: 1+\mathrm{H}-\mathrm{Nb}_{2} \mathrm{O}_{5}(?) \\
3: 1+\mathrm{H}-\mathrm{Nb}_{2} \mathrm{O}_{5} \\
3: 1+\mathrm{H}-\mathrm{Nb}_{2} \mathrm{O}_{5} \\
\mathrm{H}-\mathrm{Nb}_{2} \mathrm{O}_{5}+3: 1(?) \\
\mathrm{H}-\mathrm{Nb}_{2} \mathrm{O}_{5} \\
\mathrm{H}-\mathrm{Nb}_{2} \mathrm{O}_{5} \\
\mathrm{H}-\mathrm{Nb}_{2} \mathrm{O}_{5} \\
\mathrm{H}-\mathrm{Nb}_{2} \mathrm{O}_{5} \\
\mathrm{H}-\mathrm{Nb}_{2} \mathrm{O}_{5}+3: 1(?) \\
\mathrm{H}-\mathrm{Nb}_{2} \mathrm{O}_{5}+\left[\mathrm{L}-\mathrm{Nb}_{2} \mathrm{O}_{5}\right]+[3: 1] \\
{\left[\mathrm{L}-\mathrm{Nb}_{2} \mathrm{O}_{5}\right]+\left[\mathrm{H}-\mathrm{Nb}_{2} \mathrm{O}_{5}\right]}\end{array}$ & $\begin{array}{l}\text { Above decomp. } 3: 1 . \\
\mathrm{N}_{g 1}<1.47 \text {, below monotectic. } \\
\text { Above monotectic. }\end{array}$ \\
\hline
\end{tabular}

1 Analyzed value for $\mathrm{Nb}_{2} \mathrm{O}_{5} ; \mathrm{B}_{2} \mathrm{O}_{3}$, by difference.

2 Specimens quenched in sealed Pt tubes.

${ }_{3}^{3}$ Phases identified are listed in order of amount present at room temperature. Phases not necessarily present at the elevated temperatures are enclosed in brackets. $3: 1$ refers to $3 \mathrm{Nb}_{2} \mathrm{O}_{5} \cdot \mathrm{B}_{2} \mathrm{O}_{3}$, the most likely composition of the binary compound.

rich devitrified liquids which were mechanically separated from samples quenched from above the monotectic. When the $\mathrm{Pt}$ was removed from the quenched specimens, two distinct layers were clearly visible. The top layer appeared opalescent white and was found to be hard and glassy when ground. An index of refraction of approximately $1.51 \pm 0.01$ was determined for clear portions of some of the grains. The lower layer appeared crystalline, metallic grey in color. Under the microscope the grains were dark and very weakly birefringent. The $\mathrm{x}$-ray powder patterns showed that the top layer $\left(\mathrm{B}_{2} \mathrm{O}_{3}\right.$-rich) contained low- $\mathrm{Nb}_{2} \mathrm{O}_{5}$ with a trace of high- $\mathrm{Nb}_{2} \mathrm{O}_{5}$, whereas the lower layer $\left(\mathrm{Nb}_{2} \mathrm{O}_{5}\right.$-rich) contained appreciable high- $\mathrm{Nb}_{2} \mathrm{O}_{5}$, low $-\mathrm{Nb}_{2} \mathrm{O}_{5}$, and a small amount of " $3 \mathrm{Nb}_{2} \mathrm{O}_{5} \cdot \mathrm{B}_{2} \mathrm{O}_{3}$."

Homogeneous glasses were never obtained for any composition as the liquids devitrified on quenching. $\mathrm{X}$-ray examination showed either low- $\mathrm{Nb}_{2} \mathrm{O}_{5}$, high$\mathrm{Nb}_{2} \mathrm{O}_{5}$, " $3 \mathrm{Nb}_{2} \mathrm{O}_{5} \cdot \mathrm{B}_{2} \mathrm{O}_{3}$ ", or combinations. The low$\mathrm{Nb}_{2} \mathrm{O}_{5}$ phase corresponded to the pseudo-orthorhombic subcell of low $-\mathrm{Nb}_{2} \mathrm{O}_{5}$ found for some of the quenched liquids in the $\mathrm{Nb}_{2} \mathrm{O}_{5}-\mathrm{GeO}_{2}$ system. It had the same unit cell dimensions [1]. The metastable low-temperature niobia-type phase of hexagonal symmetry occurring in the $\mathrm{GeO}_{2}$ system was not found. 


\subsection{Compound " $3 \mathrm{Nb}_{2} \mathrm{O}_{5} \cdot \mathrm{B}_{2} \mathrm{O}_{3}$ "}

The exact composition of this compound could not be determined because of insufficient sensitivity, in this instance, of the microscopic and x-ray methods together with some uncertainty in the chemical analyses. Therefore, the $3: 1$ ratio of oxides is given as the most probable.

The compound formed readily for all compositions in the system. It melted incongruently at about 1150 ${ }^{\circ} \mathrm{C}$ to liquid and $\mathrm{H}-\mathrm{Nb}_{2} \mathrm{O}_{5}$. However, disappearance of the compound above the melting point was extremely sluggish. The compound could be detected in diminishing amounts up to the monotectic and liquidus temperatures (see table 1). Even compositions heated at $1161{ }^{\circ} \mathrm{C}$ for $68 \mathrm{hr}$ showed appreciable amounts of the compound, but the appearance of $\mathrm{H}-\mathrm{Nb}_{2} \mathrm{O}_{5}$ indicated that the temperature was above the incongruent melting point. X-ray powder diffraction data suitable for identification is given in table 2. The pattern is of low symmetry with regions of closely spaced low-intensity peaks and could not be indexed.

TABLE 2. X-ray powder diffraction data $\left(\mathrm{CuK}_{\alpha}\right.$ radiation) for the compound " $3 \mathrm{Nb}_{2} \mathrm{O}_{5} \cdot \mathrm{B}_{2} \mathrm{O}_{3}$ "

\begin{tabular}{|c|c|c|c|}
\hline$d^{\prime}$ & $I / I_{0}$ & $d$ & $I / I_{0}$ \\
\hline$\AA$ & $\%$ & $\AA$ & $\%$ \\
\hline${ }^{*} 14.4(\mathrm{~b})$ & 4 & 2.434 & 3 \\
\hline 7.16 & 7 & 2.389 & 6 \\
\hline 6.93 & 6 & 2.369 & 4 \\
\hline 4.774 & 34 & 2.337 & 3 \\
\hline 4.741 & 27 & 2.302 & 17 \\
\hline 3.770 & 71 & ${ }^{*} 2.094(\mathrm{~b})$ & 3 \\
\hline 3.584 & 90 & 2.049 & 26 \\
\hline 3.553 & 100 & 2.033 & 27 \\
\hline 3.170 & 12 & 1.9125 & 33 \\
\hline 2.864 & 6 & ${ }^{*} 1.7918(b)$ & 5 \\
\hline 2.833 & 10 & 1.7746 & 10 \\
\hline 2.773 & 57 & 1.7697 & 10 \\
\hline${ }^{*} 2.730$ (b) & 7 & 1.6995 & 22 \\
\hline 2.675 & 9 & 1.6874 & 21 \\
\hline 2.576 & 6 & 1.6842 & 23 \\
\hline 2.520 & 7 & ${ }^{*} 1.5838(\mathrm{~b})$ & 6 \\
\hline 2.506 & 9 & 1.5769 & 16 \\
\hline 2.473 & 4 & & \\
\hline
\end{tabular}

${ }^{*} \mathrm{~b}=$ broad peak

\subsection{Application to Liquid Immiscibility Theory}

The niobium borate and niobium silicate systems both contain large regions of immiscibility, whereas the niobium germanate system shows complete liquid miscibility. Thus, as previously discussed [1], the niobium cation possesses a critical ionic field strength for the formation of two liquids with the glass-forming cations.

With data on immiscibility in the niobium borate and silicate systems, it is possible to test and to extend the principles of immiscibility developed mostly from data on the divalent cations to the cations of high charge. A fundamental consideration in the structural approach is the average number of oxygens associated with a modifier cation, in the modifier-rich liquid. Once this value is known, statement of the nominal composition is a routine calculation. The oxygen-volume method, discussed in previous publications $[3,6]$ is an attempt to make a reasonable estimate of this number.

TABLE 3. Calculated composition of modifier-rich liquids, in \#0/Nb and in mole percent $\mathrm{Nb}_{2} \mathrm{O}_{5}$, based on the oxygen-volume method [6] and two values both for the niobium separation and the volume of space occupied by $0^{-2}\left(\mathrm{~V}_{0}\right)$.

\begin{tabular}{|c|c|c|c|c|c|c|c|c|}
\hline \multirow{4}{*}{ System } & \multirow{3}{*}{\multicolumn{2}{|c|}{ Experimental }} & \multicolumn{6}{|c|}{ Calculated from $\mathrm{Nb}-\mathrm{Nb}$ separation: } \\
\hline & & & \multicolumn{4}{|c|}{ of $4.18 \AA[2(1.40+0.69)]$, and } & \multirow{2}{*}{\multicolumn{2}{|c|}{$\begin{array}{c}\text { of } 4.08 \AA \text {, and } \\
V_{0}=19 \AA^{3}\end{array}$}} \\
\hline & & & \multicolumn{2}{|c|}{$V_{0}=17 \AA^{3}$} & \multicolumn{2}{|c|}{$V_{0}=19 \AA^{3}$} & & \\
\hline & $\mathrm{Nb}_{2} \mathrm{O}_{5}$ & $\# 0 / \mathrm{Nb}$ & $\# 0 / \mathrm{Nb}$ & $\mathrm{Nb}_{2} \mathrm{O}_{5}$ & $\# 0 / \mathrm{Nb}$ & $\mathrm{Nb}_{2} \mathrm{O}_{5}$ & $\# 0 / \mathrm{Nb}$ & $\mathrm{Nb}_{2} \mathrm{O}_{5}$ \\
\hline $\begin{array}{l}\mathrm{Nb}_{2} \mathrm{O}_{5}-\mathrm{B}_{2} \mathrm{O}_{3} \\
\mathrm{Nb}_{2} \mathrm{O}_{5}-\mathrm{SiO}_{2}[2]\end{array}$ & $\begin{array}{r}\text { Mole \% } \\
65.7 \\
47.5\end{array}$ & $\begin{array}{l}3.28 \\
3.61\end{array}$ & $\begin{array}{l}4.18 \\
4.18\end{array}$ & $\begin{array}{r}\text { Mole \% } \\
47.2 \\
37.4\end{array}$ & $\begin{array}{l}3.72 \\
3.72\end{array}$ & $\begin{array}{r}\text { Mole \% } \\
55.1 \\
45.0\end{array}$ & $\begin{array}{l}3.45 \\
3.45\end{array}$ & $\begin{array}{r}\text { Mole\% } \\
61.1 \\
51.2\end{array}$ \\
\hline
\end{tabular}

Table 3, column 3 gives the number of oxygens per modifier cation calculated directly from the experimentally determined composition of the modifier-rich liquid. For example, in the $\mathrm{Nb}_{2} \mathrm{O}_{5}-\mathrm{B}_{2} \mathrm{O}_{3}$ system:

$$
\# 0 / \mathrm{Nb}=\frac{5 \times 0.657+3(1.00-0.657)}{2 \times 0.657}=3.28
$$

Similarly, in the $\mathrm{Nb}_{2} \mathrm{O}_{5}-\mathrm{SiO}_{2}$ system:

$$
\# 0 / \mathrm{Nb}=\frac{5 \times 0.475+2(1.00-0.475)}{2 \times 0.475}=3.61 .
$$

As a first approximation the number of oxygens per cation may be considered independent of the glassforming cation. Using the data in column 3 for one of the systems, it is possible to calculate the composition of the modifier-rich liquid in the other system. The calculation yields 57.5 mole percent $\mathrm{Nb}_{2} \mathrm{O}_{5}$ in the borate system, ${ }^{2}$ using the 3.61 value, and 56.2 mole percent $\mathrm{Nb}_{2} \mathrm{O}_{5}$ in the silicate system, using the 3.28 value. The calculated value in the borate system is about 8 mole percent low, that in the silicate system about 9 mole percent high. As will be shown later, this seemingly large discrepancy is due to the sensitivity of the calculations in the niobium oxide systems.

Table 3 (columns 4-7) lists the calculated \#0/Nb and the corresponding mole percent $\mathrm{Nb}_{2} \mathrm{O}_{5}$, based on the oxygen-volume method using a $\mathrm{Nb}-\mathrm{O}-\mathrm{Nb}$ bond angle of $180^{\circ}$ and Ahrens' ionic radii for $\mathrm{Nb}^{+5}$ and $\mathrm{O}^{-2}$. In the original papers on immiscibility the value of $17 \AA^{3}$ was taken as the average volume of space occupied by an oxygen atom in the modifier-rich liquid.

\footnotetext{
${ }^{2}$ Nominal oxide formula $=\frac{\mathrm{Nb}_{2} \mathrm{O}_{5} \cdot(2 \times 3.61-5)}{3} \mathrm{~B}_{2} \mathrm{O}_{3}=\mathrm{Nb}_{2} \mathrm{O}_{5} \cdot 0.74 \mathrm{~B}_{2} \mathrm{O}_{3}(57.5$ mole $\%$
} $\mathrm{Nb}_{2} \mathrm{O}_{5}$ ). 
An analysis of the experimental data in about 40 borate and silicate systems showing immiscibility [7] gives an empirical value of about $19 \AA^{3}$. Therefore, in table 3 calculations are given for both oxygen values. It may be seen that for the $19 \AA^{3}$ value in the silica case agreement is good between the calculated mole percent $\mathrm{Nb}_{2} \mathrm{O}_{5}(45.0)$ and the experimental one (47.5). In the borate case the calculated value is 11 mole percent low.

For the case of small highly charged modifier cations, such as niobium, the calculations are very sensitive to cationic radius because a small difference in separation has a large effect on the calculated number of oxygen per cation. For example, using a separation of only $0.1 \AA$ less than 4.18 , will increase the calculated value by about 6 mole percent (column 9 ); and the calculated number of oxygens per niobium (column 8) is intermediate between 3.28 and 3.61, the experimentally determined values for the borate and silicate systems, respectively. Gatehouse and Wadsley [8], from a crystal structure determination of high- $\mathrm{Nb}_{2} \mathrm{O}_{5}$, have concluded that in $\mathrm{Nb}_{2} \mathrm{O}_{5}$ the average ionic radius of $\mathrm{O}^{2-}$ is $1.40 \AA$ and that of $\mathrm{Nb}^{5+}, 0.59 \AA$. The latter value is $0.1 \AA$ less than Ahrens' ionic radius.

\section{Summary}

The phase equilibrium diagram for the system $\mathrm{Nb}_{2} \mathrm{O}_{5}-\mathrm{B}_{2} \mathrm{O}_{3}$ was constructed from "quenching" data on nine selected compositions. Solidus and liquidus values were determined by examination of the samples with the binocular and polarizing microscopes and x-ray powder diffractometry.

The system was found to contain one binary compound, " $3 \mathrm{Nb}_{2} \mathrm{O}_{5} \cdot \mathrm{B}_{2} \mathrm{O}_{3}$ ", which melts incongruently at about $1150{ }^{\circ} \mathrm{C}$, some 200 below a monotectic. The liquidus is characterized by a large two-liquid region, at $1352{ }^{\circ} \mathrm{C}$, extending from about 10 mole percent $\mathrm{Nb}_{2} \mathrm{O}_{5}$ to 65.7 mole percent $\mathrm{Nb}_{2} \mathrm{O}_{5}$.
Experimental limitations precluded an unequivocal statement of the composition of the " $3 \mathrm{Nb}_{2} \mathrm{O}_{5} \cdot \mathrm{B}_{2} \mathrm{O}_{3}$ " compound. Above the incongruent melting point its decomposition to $\mathrm{H}-\mathrm{Nb}_{2} \mathrm{O}_{5}$ and $\mathrm{B}_{2} \mathrm{O}_{3}$-rich liquid is sluggish. X-ray powder data suitable for identification is listed.

In the modifier-rich liquid in the immiscibility region, an average of 3.28 oxygen are associated with each $\mathrm{Nb}$ atom, as compared to 3.61 for the corresponding silicate system. Calculation of the composition of the modifier-rich liquid, by the oxygen-volume method, gives acceptable agreement with the experimentally determined value, considering that for cations of large valence the calculation is sensitive to small changes in the parameters.

\section{References}

[1] E. M. Levin, J. Res. NBS 70A (Phys. and Chem.), No. 1, 5-10 (1966).

[2] M. Ibrahim and N. F. H. Bright, J. Am. Ceram. Soc. 45 [5], 221-22 (1962).

[3] (a) E. M. Levin, C. R. Robbins, and J. L. Waring, J. Am. Ceram. Soc. 44 [2], 87-91 (1961).

(b) E. M. Levin and C. L. McDaniel, J. Am. Ceram. Soc. 45 [8], 355-60 (1962).

[4] (a) J. L. Waring and R. S. Roth, J. Res. NBS 69A (Phys. and Chem.), No. 2, 119-29 (1965).

(b) R. S. Roth and J. L. Waring, J. Res. NBS 65A (Phys. and Chem.), No. 4, 337-44 (1961).

[5] T. J. Rockett and W. R. Foster, J. Am. Ceram. Soc. 48 [2], 75-80 (1965).

[6] E. M. Levin and S. Block, J. Am. Ceram. Soc. 40 [3], 95-106; [4], 113-18 (1957).

[7] E. M. Levin, Structural interpretation of immiscibility: parts IV, V, and VI, in preparation.

[8] B. M. Gatehouse and A. D. Wadsley, Acta Cryst. 17 [12], 1545-54 (1964).

(Paper 70A1-382) 